DOE/ER/45254--7

DE92 014841

STRUCTURE AND DYNAMICS IN LOW-DIMENSIONAL GUEST-HOST SYSTEMS

Progress Report

for period June 1, 1990 through May 31, 1992 (yr. 3)

Professor John E. Fischer, MSE

Department of Materials Science \& Engineering, School of Engineering and Applied Science, University of Pennsylvania, Philadelphia

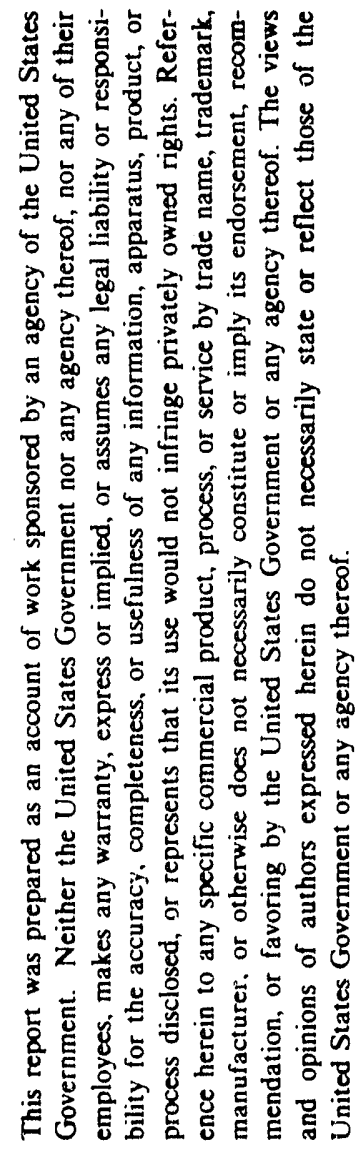

Apri1 19992

prepared for:

THE UNITED STATES DEPARTMENT OF ENERGY

Agreement no.: DE-FG02-85ER-45254 


\title{
STRUCTURE AND DYNAMICS IN \\ LOW-DIMENSIONAL GUEST-HOST SYSTEMS
}

\author{
John E. Fischer \\ Materials Science Department \\ University of Pennsyluania \\ Philadelphia PA 19104-6272
}

\section{INTRODUCTION}

New synthetic materials continue to be discovered at a rapid rate. Many of these can be broadly described as guest-host systems, in the sense that a range of compositions is accessable by selectively inserting heteroatoms or molecules into the interstitial sites in an otherwise pure starting material. The premier examples are layer intercalate's (graphite. transition metal di- and trichalcogenides, silicate clays) and doped polymers (notably polyacetylene). With is somewhat broader definition of intercaiation, one might include the high- $T_{c}$ cuprate superconductors (variable oxygen and alkaline earth concentrations), ion-exchanged beta-alumina and related defect oxides. and alkali metal-doped buckminsterfullerene $\left(C_{60}\right)$. The interest in these material families for energy applications is directly attributable 10 the guest-in-a-host feature, either by e.spioiting guest ion mobility in electrochemical devices or by tuning/optimizinor properties via control of guest concentration and sublattice structure.

It is becoming increasingly apparent that great advantages are to be gained by considering these classes of complex materials globally, as an alternative tc focussing on one family at a time. Important examples of beautiful unifying themes have been identified in some cases, and these are ripe for further exploitation. Our work in the ' 70 's on graphite intercalation compounds evolved naturally into related experiments on transition metal dichalcogenide intercalates and doped polyacetylene. This background in guest-host systems enabled us to make a major impact on the new field of "buckyball" materials and their dofed derivatives. (A brief foray into cuprates was not enormously useful and has been dropped.)

This proposal is for a 3-year renewal of the current grant of the same title, DE-FG02-86ER45254, for which $\$ 827.568$ has been awarded ove، the 6 -year period $6 / 1 / 86-5 / 31 / 92$. Part II of this document is a progress report covering the first 25 months $(6 / 89$ to $i / 91)$ of the present 3 -year period. Part IV describes the proposed research $6 / 1 / 02-5 / 31 / 95$. Selected reprints and manuscripts in process are attached as appendices.

\section{HIGHLIGHTS OF CURRENT GRANT}

\section{A. PREAMBLE}

From the previous 3year renewal proposal, written in mid-1988:

".... Research on these materials has proceeded independently, with little effort devoted to exploring their commonalities. It has been amply demonstrate.1 that they all exhibit unusual features in their static crystal structures. lattice vibrational properties and/or guesi diffusion dynamics. It thus seems evident that opportunities exist to transfer knowledge from the more mattre subtields to the newer ones, in order to address crucial problems in structure and dynamics...."

".... We have been active since 1975 in the study of graphite intercalation compounds, in which the most dramatic structural feature is the staging phen mmenon - a sequence of $1-D$ superlattices of alternating host and guest layers whose periodicity and perfection vary with all the thermodynarnic variables. Staging is thus intimately involved in the above issues. Major progress has been made in the (previous) grant period in understanding staging in terms of competing interactions (inter- vs. intralayer, guest-guest vs. guest-host etc.), to the extent that a unifying microscopic picture is emerging....."

"The principal experimental tools are $x$-ray and neutron scattering, using in-house facilities, the HFBR and NSLS at Brookhaven, and the ILL in Grenoble as appropriate. (We are making a conscious effort to increase the supply if $P l_{1} D$ 's trained in neutron scattering.) Jample materials are either grown in-house or provided through collaborations with other groups." 


\section{B. GRAPHITE INTERCALATION COMPOUNDS}

\section{Structure and Dynamics in $C_{B} A_{B} F_{5}$}

We used elastic, inelastic and quasielastic neutron scattering to study the temperature-dependent structure anc. dynamics in the prototype stage-1 acceptor compound $C_{B} A s F_{5}$. It is well-known that $A s F_{5}$ dissociates upon intercalation; the diversity of constituents in he intercalate layer guarantees a rich but complex phase diagram. HendrichTeller broadening of ( $\mathrm{OL}$ ) profiles indicate a tendency to form stage-2 galleries at low $\mathrm{T}$, so the average out-of-plane intercalate density must decrease with decreasing $T$. The implied converse dependence of in-plane density is revealec in a complicated sequence of ordered in-plane structures, one of which becomes denser with decreasing $T$ but never locks in to the graphite periodicity. So:ne of the molecular motions are slow enough (even at low $T$ ) to observe quasielastic line broadening (or QENS) with our triple-axis spectrometer. Unlike the alkali GIC's, we see no finite-ener's!' peaks associated with discrete intercalate-derived phonon modes, not even down to 10K. The Q-dependence of ihe quasi-elastic width exhibits structure on top of an overall $Q^{2}$ dependence. We associate the latter with free-particle molecular diffusion, while the structurs is likely due to jump diffusion or rotation.

\section{Jump Diffugion and Phonon DOS in Stage-2 Li-GIC's}

In this project we exploited the different in-plane densities and T-dependent structures of $\mathrm{LiC}_{2}$ and $\mathrm{LiC}_{16}$ to study jump diffusion and in-plane phonon spectra versus stage and long- and short-range order, taking the $L i C_{b}$ results as $i$ point of departure. We found no QEN!S in $L_{i} C_{12}$ for $T<T_{m}=500 \mathrm{~K}$, while the T-dependent quasielastic broadening energy $\Gamma(Q)$ for $L i C_{16}$ is consistent with nearest-hexagon jumps by incoherent scatterers over a barrier 0.55 ev. half the value obtained in the ordered phase of $\mathrm{LiC}_{6}$.

For $\mathrm{T}>500 \mathrm{~K}, \mathrm{LiC}_{12}$ is dilute stage-1 while $\mathrm{LiC}_{16}$ is less-dilute stage-2; surprisingly, both $\Gamma(Q)$ 's imply a jump vector $L$ close to 4.92 A (third-neighbor sites) and a much smaller barrier energy. A jump vector this long is highly unlikely since essentially all the sites are acessable to a jump-diffusing particle in both compounds. We suspect that this inconsistency results from the use of oversimplified models for the QENS broadening, either the neglect of spatial and temporal correlations in the jump process itself, or the assumption that the QENS is dominated by incoherent scattering from Li. Q-integrated inelastic spectra reveal structure in the phonon DOS which disappears above $T_{m}$ in $L_{i} C_{12}$; the heavy alkali analogs (e.g. $R L_{1} C_{24}$ ) behave quite differently, presumably due to a domain-discommensuration lattice which characterizes the low-T crdered phase of heavy alkali compounds.

\section{STAGING TRANSITIONS IN LL- AND Ag-TiS,}

Graphite intercalation compounds transform at high pressure to higher stages with greater in-plane densities. Tliest transformations are driven by local elastic distortions which favor the creation of dense islands, which in turn correlate in the c-direction to give well-ordered staging superlattices. The entire process can be modelled in a continuum theory which accounts for the host layer elasicic properties. The pressure response using different intercalates depends only on the c-axis component of the local distortion since the in-plane "healing length" is purely a property of the hosi layers. Other groups have developed generic continuum models for elastic interactions, the implication being that. staging transitions for any intercalate in any host lattice may be thus analyzed merely by scaling to the intercalate size and the host layer bending modulus.

Our results on ITMD's show that tne continuum approach breaks down for moderately stiff host lattices. Dilute stage-1 $L_{0.5} \mathrm{TiS}_{2}$ remains stage-1 up to $55 \mathrm{kbar}$ while a similar Ag compound exhibits a combined staging/polytype transition at very low pressure. It is difficult to reconcile a large difference in threshold pressures within the continuum model, since the healing length (which controls the magnitude of the in-plane attractive interaction) is independent of the intercalate radius. On the other hand, our rest 1.5 can be rationalized by accounting for the different intercalate radii compared to the volume of a tetra sedral interstitial site. Li is small enough that a reduction in specific density per intercalate with increasing pressure is r.ot required. The c-axis strain can easily be accommodated just by compressing the van der IVaals gallery. Li easily "hi.jes" in the interstitial site up to a predicted pressure of $70 \mathrm{kbar}$, at which point a further volume reduction requires creation of dense islands and empty regions. On the other hand, Agt completely fills the tetrahed. 1 interstices already at 1 atm., thus the phase transition begins at very low $P$ (see $A p p e n d i x A$ ). 


\section{STRUCTURE AND DYNAMICS OF CONDUCTING POLYMERS}

\section{Staging in Doped Polymers}

In principle the low- $T$ dilute phises in these materials should consist of $2 D$ superlattices of fully-occupied ID channels, analogous to the 1D layer sequences ("stages") of densely-filled 2D galleries in GIC's. We previously calculated a mean-field phase diagram a la Safran, mainly as a guide to the experiments. Using in situ x-ray diffraction and electrochemical doping, we found two stages in $\left[(C H)_{y}\right]_{x}$ with y (or $\left.V_{o c}\right)$ as independent variable. The results confirm directly for the first time that anomalies in macroscopic quantities vs. $V_{o c}$ do indeed signal first-order structural transitions. Detailed analysis shows that the polymer chain setting angle goes through several stepwise rotations as the channels are "prepared" to accept the alkali intercalant (see Appendix B).

\section{Broken Symmetry Intercalation Lattices}

The lattice symmetry of polymer intercalation compounds depends fundamentally upon the size of the intercalate. unlike the 1-D staging structures in layer intercalates. In particular, the formation of quasi-1D dopant channels imposes rotations of the host chains about their long axes such that small alkalis $(\mathrm{Li}, \mathrm{Na})$ induce a triangular superlattice while large alkalis (K, Rb, Cs) force larger channels with square 2D symmetry. We have performed detailed analysis of high-quality $\mathrm{x}$-ray diffraction measurements on K-doped $(\mathrm{CH})_{x}$ and Cs-doped (poly)paraphenylene-vinylene (PPV) which demonstrate that the simple symmetries previously assumed are broken by rotations and translations of the polymer chains, presumably originati.ag in some new interchain interactions. Steric arguments can be applied to predict similar phenomena in other systems, with implications for both the phase diagrams and 3-D band structure calculations. This topic is being studied in collaboration with Prof. M. Winokur, U. Wisconsin (see Appendix C).

\section{Structure and Dynamics in Cs-doped Polyacetylene}

The $x$-ray profile of heaviily doped $\left[(\mathrm{CH}) \mathrm{Cs} s_{y}\right]_{x}$ was analyzed on the basis of three-dimensionally ordered intercalate channels. Fifteen peaks representing 27 unique reflections are well-represented (positions and intensities) by a tetragonal pseudo-cell with $\mathrm{a}=\mathrm{b}=\mathfrak{c} .093 \dot{A}, \mathrm{c}=7.950 \dot{A}$ containing $24(\mathrm{CH})$ and $4 \mathrm{Cs}$ units $(\mathrm{y}=0.167)$. The Cs and $(\mathrm{CH})$ sublattices are actually incummensurate along $c$, with 6.46 undistorted $(\mathrm{CH})$ units per Cs implyin $\%$ y $=$ 0.15. Reflections with non-zero $L$ are significantly less intense than calculated neglecting the Debye-Waller factor. indicating large amplitude $c$-axis thermal motion of $\mathrm{Cs}$ ions. The fractional linear in-plane dilation upon replacing $\mathrm{K}$ with $\mathrm{Cs}$ is within $20 \%$ of the corresponding value in graphite intercalates (see Appendix D).

\section{Conductivity and Cell Potential in $\left[(C H) K_{y}\right]_{x}$}

A high-resolution quasi-equilibrium in situ study of electrical conductivity and open circuit voltage $V_{\text {oc }}$ during potassium doping and dedoping, of an oriented $(C H)_{x}$ electrode has been performed. The goal was to identify anomalies which might be associated with subtle structural transitions, in order to guide future detailed x-ray experiments. Features in $d V_{o c} / d y$ and $d \sigma / d y^{\prime}(\mathrm{y}=\mathrm{K}$ mole fraction) found previously at $\mathrm{y}=0.06,0.12$ and 0.15 were confirmed and correlated with recent $x-$ ray and esr results and with a model of staging via intercalation channels. We also found new features at $y=0.03$ and 0.18 , the former coinciding with a similar esr feature. We propose that these are signatures of structural effects which do not involve major changes in lattice constants or unit cell symmetries. We tentatively assign the $y=0.03$ feature to a metastable "dilute stage- $2^{n}$ structure by analogy to graphite intercalates. Three phases with the same stage-1 channel structure are inferred from the observation of three plateaus in $V_{o c}(y)$ in the range $0.10<y<0.17$ upon dedoping. A maximum $\sigma=17500 \mathrm{~S} / \mathrm{cm}$ is found near $\mathrm{y}=0.12$ during the first doping cycle. After successive cycles $\sigma_{\max }$ decreases, the maximum attainable $y$ also decreases, and the overall form of $V_{o c}$ vs. $y$ approaches the ideal behr:vior for an intercalation electrode exhibiting first-order phase transitions (see Appendix E).

\section{Polarized Vibrational Density of States}

It is now well-established that the novel physics of conjugated polymers results from strong electron-phonon and/or electron-electron interactions. The conceptual state-of-the-art is the Su-Schrieffer-Heeger Hamiltonian, which treats electron-phonon coupling within a single chain at the level of BCS theory - a single empirical number akin to the Mac.Millan $\lambda$ parameterizes the whole problem. Now that the importance of 3-D effects is becoming important, one 
would like to have a better model which incorporates the actual phonon spectrum and includes inter-chain vibrationil modes.

-Experimental results on vibrations are limited to a few zone-center intrachain modes (Raman, IR): theorists haw. yet to attempt a 3-D lattice dynamics calculation. More detailed experiments are frustrated by the linited quality anil quantity of available samples - a complete phonon dispersion study via coherent inelastic neutron scattering woul.l require several grams of deuterated material with a c-axis mosaic at least twice as good as the best available. Is I first step towards this ultimate goal. we have developed a technique to obtain polarized densities of vibrational statifrom incoherent inelastic neutron scattering. In this technique the finite mosaic is exploited to Q-integrate the ener"y!dependent scattering from hydrogen motions. A simple rotation of the sample allows to separate the two principal polarizations. which gives for the first time information about inter-chain vibrations and 3-D effects.

A first 4-day run at the Institut Late-Langevin reactor yielded results for polyacetylene and polyaniline at seviral temperatures. We proved the validity of the technique by verifying the polarization of previously-known modes. Mor" importantly: we identified a number of new features, in particular a mode at $3 \mathrm{meV}$ polarized along the chains. Thip best candidate for this very soft modis is the out-of-phase longitudinal motion of the two inequivalent chains/coll. This mode will be very sensitive to changes in interchain interactions with doping, and may also serve as a rather direct probe of topological defects (solitons, polarons). Analysis of polyaniline data (in progress) shows a mode at l: mel' which may turn out to be the rir.g torsion mode responsible for thermochromism (see Appendix F).

\section{Crystal Structure of trans-Polyacetylene Revisitied}

New $x$-ray diffraction data on high-quality trans $-(C H)_{x}$ shows that the bond alternation is out of phase on adjacenl chains, and therefore the correct space group is $P 2_{1} / n$. Previous attempts to resolve this question by analyzing only the $(00 \mathrm{~L})$ intensities have yielded ambiguous results; all of the previously-published diffraction experiments show some (001) intensity which is not allo'ved in $P 2_{1} / n$. By analyzing individual off-axis peak intensities. we were $\left.a b\right)$. to show unambiguously that the bulk: $3-\mathrm{D}$ structure is inconsistent with $P 2_{1} / a$ (in-phase bond alternation). and therefore the weak. sample-dependent (001) intensity results from uncorrelated local defect regions with in-phas. bond alternation on adjacent chains. This observation may provide a clue for explaining the irreproducibility if extremely high conduvtivities claimed for the various "new, improved" forms of $(C H)_{x}$ which have recently been reported.

We were able to rule out the possibility of bulk phase coexistence of $P 2_{1} / a$ and $P 2_{1} / n$ structures as follows. Considering the (00L) intensities alone implies the existence of $20 \%$ of $P 2_{1} / a$ phase in our sample. while the ratio of off-axis (021) and (011) intensities place an upper limit of only 4\%. The (001) intensity must therefore be associated with a local defect. for example short hain segments which correlate with neighboring longer chains in the in-phase configuration. It would be interesting to perform a comparative study of various kinds of $(C H)_{x}$ to find out if a variable density of such short-chain defects can be correlated with differences in macroscopic properties. e.g. conductivity aftır iodine doping (see Appendix G).

\section{E. SOLID PHASES DERIVED FROM BUCKYBALLS}

\section{Introduction}

A new crystalline form of pure carbon was reported less than a year ago. Unlike diamond and graphite. this now material ("buckminsterfullerite") is constructed from soccer ball-shaped 60-atom molecules ("buckyballs") which are atomic-scale analogs of Fuller's geodesic domes. Chemists are claiming that these highly stable yet modifiable molecules will generate an entirely new class of organic compounds. The implications for materials science may also be far-reaching. Speculations hav" been made concerning applications as lubricants, selective tuneable catalysts and electrochemical energy storage miterials. Additional excitement has been generated by the recent discoveries of metallic and superconducting behavio: in alkali metal-doped fullerites.

The a vailability of a broad range of expertise and central facilities under one roof made it possible for LRS.M faculty and staff to quickly make a major impact on this rapidly growing field. Our interest was intially stimulated by a visit from Prof. R. Smalley (one of the co-discoverers of $C_{60}$ ) in October 1990, which revealed several obvious connections between his beloved buckyballs and our ongoing research: the complementarity between liquid and plastic crystals (i.e. orientational vs. positional long-range order); possible analogies between layer intercalates, doped conjugated polymers and doped $C_{60}$; and the prosp.ect for rapid advancement through close coupling among synthesis, experiment. simulations and theory. We began meeting once a week to plan experiments, share ideas etc.. and produced our first purc $C_{\text {no }}$ in late December. As of this writing 19 papers have been published or submitted by Penn faculty. The LRS.II 
has also established an international electronic preprint alert service. and is collaborating with Smalley to maintain his bibliographic data base. We also hosted a 2-day workshop in early August. focussing on condensed phases and superconductivity. A large number of external collaborations have already taken shape. including proposals will groups in France and Israel. The highlights of DOE-funded achievements in this exploding field are summarizol below.

\section{Structure, Bonding and Dynamics in Solid $C_{60}$}

A closely-coordinated program of $x$-ray and neutron scattering, model calculations and molecular dynamics sim. ulations. revealed the salient features of the solid $C_{60}$ lattice. Pure $C_{60}$ at $300 \mathrm{~K}$ is a face-centered "plastic" crystal with long-range positional order (>1000 $\AA$ correlation length) but no molecular orientational order. the converse is nematic liquid crystals. It transforms to an orientationally-ordered simple cubic structure below $249 \mathrm{~K}$, as observed hy $x$-ray diffraction and scanning calorimetry. Molecular dynamics simulations using a potential derived from graphili. gives the correct lattice constant and repruduces the observed orientational freezing below $200 \mathrm{~K}$. The (isotropic) line:ir compressibility is the same as the c-axis compressibility of graphite. consistent with van der Waals intermoleculiur bonding; molecular dynamics and lattice energy calculations agree with this experiment (see Appendices $H$ and .1 ).

\section{Alkali Metal-Doped $C_{60}$}

Compound synthesis, x-ray scattering, molecular dynamics (Prof. .I. Klein) and electron spectroscopy (Prof. II. Plummer) have been applied to study che binary alkali metal- $C_{\text {no }}$ phases. $M_{x} C_{\text {ño }}$. in order to understand the metalli:

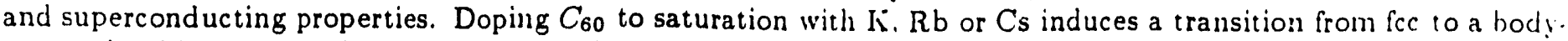
centered cubic compound of ideal corrposition $M_{6} C_{60}$. This implies a competition between elastic and electrostatir energies as the metal concentration is increased from zero. which should lead to an interesting phase diagram wirl. some features common to layer intercalates and doped polymers (see below). Molecular dynamics simulations clearls reveal the transition from fcc to bcc with increasing metal concentration. the crossover occurring at $x=3$ which $\mathrm{i}$ currently believed to be the only superconducting phase. Electron spectra are consistent with the existence of onl: three distinct phases $(x=0,3$ and 6$)$. !Hgh-resolution photoenission shows a clear Fermi energy threshold over a lar range $0.5<x<5$, while the $C 1$ abscrption spectra vs. $x$ can be interpreted as linear combinations of single-phas. spectra with $x=0.3$ and 6 . Photoemission and molecular dynamics give values for the density of states at the Fermi energy and the low-energy librational tiequency respectively. Assuming that the latter is responsible for the pairin! interaction. BCS theory gives a coupling constant $\lambda=1.06$. intermediate between weak-and strong-coupling limit: (see Appiendix Ki).

\section{Fluorinated Fullerenes}

We have: shown that $C_{60}$ and $C_{70}$ take up fluorine under mild conditions (300K. 100-300 torr) to yield partially. crystalline tan to yellow powdered solids. In contrast to the alkali-doped phases. the fluorinated material cannot be described as analogous to intercalation compounds. Infrared absorption shows clear evidence for C-F bonds. and mass speciurometry under a variety of source conditions shows stable species with $F / C_{\text {bio }}$ ratios ranging from 22 to 52 . Weight uptake gives an average compissition near $C_{60} F_{36}$, and indeed the mass distribution shows a maximum n $\mathrm{nr}$ this value. X-ray diffraction analysis grves an fcc cell with $\mathbf{a}=17.0$. . consistent with close-packed spheres of radius about 1 \& llarger than $C_{60}$ ("hairyballs"). Work is in progress to isolate $C_{60} F_{35}$. to achieve complete fluorination up to $C_{60} F_{50}$, and to selectively fluorinate one or two $C$ sites using milder fluorinating agents. This work is being done in collabora:ion with Prof. Henry Selig (Il ebrew University) who spent the summer at Penn and trained one of my students to carry out fluorine doping reactions (see Appendix L). 


\section{F. PUBLICATIONS CITING DOE SUPPORT: 1080-1001}

1. "X-ray, Neutron and Electron Diffraction Study of the Incommensurate Structure in Single Crystals of Superconducting $\mathrm{Bi}_{2.2} \mathrm{Sr}_{1.9} \mathrm{CaCu}_{2} \mathrm{O}_{8+x}$ ", J. E. Fischer, P. A. Heiney, P. K. Davies and D. Vaknin, Phys. Rev. B39. 2Tj'2 $(1080)$.

2. "In Silu Study of Staging Disorder i., Cesium-Intercalated Graphite", V. B. Cajipe. P. A. Heiney and J. E. Fischer". Phys. Rev. B39, 4374 (1989).

3. "High-Temperature Lattice-Liquid In-Plane Structure of $L i C_{b}$ : A . Veutron Scattering Study". H. J. Kim.

A. Magerl. J. L. Soubeyroux and J. E. Fischer, Phys. Rev. B39. 4670 (1980).

4. "Structural Characterization of a Naw Polyacetylene", D. Djurado. J. Ma. N. Theophilou and J. E. Fischer.

Synthetic Metals 30, 395 (1989).

5. "Antiferromagnetism in $\mathrm{Ca}_{0.85} \mathrm{Sr}_{0 .: 5} \mathrm{CuO}_{2}$, the Parent of the Cuprate Family of Superconducting Compounds".

D. Vaknin. E. Caijnol, P. K. Davies, J. E. Fischer, D. C. Johnston and D. C. Goshorn, Phys. Rev. B30. 9122 (1989).

6. "Highly Conducting Polyanilines and Polyacetylene/Polyaniline Composites". N. Theophilou. A. G. MacDiarmid.

D. Djurado, J. E. Fischer and A. J. Epstein, in Springer Series in Solid State Science 01. 29 (1980).

$\tau$. "Staging in Potassium-doped Polyacetylene: In-Situ X-ray Diffraction", D. Djurado. J. E. Fischer. P. A. Heinel.

J. Ma. N. Coustel and P. Bernier, Synthetic Metals 34, 683 (1990).

8. "X-ray Structure of Cs-doped Polyacetylene", J. Ma D. Djurado. J. E. Fischer. N. Coustel. P. Bernier.

Physs. Rev. B41, 2971 (1990).

9. "Structure and Dynamics of $C_{8} A s F_{5}$ : A Neutron Scattering Study", D. Vaknin. J. E. Fischer. D. Djurado. J. Ma and J. W. Milliken, Synthetic Metals 54,353 (1990).

10. "Spin-Lattice Relaxation of ${ }^{8} \mathrm{Li}$ in $\mathrm{LiC}_{12}$ at Low Temperatures", A. Schirmer. P. Heitjans, IV. Faber and

J. E. Fischer, Synthetic Metals 34, 58!) (1990).

11. "Intercalated Graphite Neutron Optical Components: Low-Mosaic Acceptor Compounds with Large d-Spacings"

J. E. Fischer, J. W. Milliken, A. Mage:!l, H. J. Kim and A. W. Moore. Nucl. Inst. Methods A300. 20T (1991).

12. "High-Resolution Study of Conductivity and Cell Potential vs. Doping Concentration in Potassium-Doped

Polyacetylene: Correlation with Structural Transitions", N. Coustel. P. Bernier and J. E. Fischer. Phy's. Rer. B43. $314 i(1991)$.

13. "New Results on the X-ray Structure of Trans- $(\mathrm{CH})_{\boldsymbol{x}}$ and its Alkali Metal Intercalation Compounds".

J. E. Fischer, P. A. Heiney and J. Ma, Synthetic Metals 41-43. 33 (1991).

14. "Polyaniline Films and Fibers: Pre'erred Orientation, Morphology and the Crystal Structure of Emeraldine Base".

J. E. Fischer, X. Tang, E. M. Scherr, \%. B. Cajipe and A. G. MacDiarmid, Synthetic Metals 41-43. 661 (1991).

15. "Polyaniline: Oriented Films and Fibers", E. M. Scherr. A. G. MacDiarmid. S. K. Manohar. J. G. Masters. I. Sun. X. Tang, M. A. Druy, P. J. Glatkcwski, V. B. Cajipe, J. E. Fischer, K. R. Cromack. M. E. Josefowicz.

J. II. Ginder, R. P. McCall and A. J. Epstein, Synthetic Metals 41-43, 735 (1991).

16. "Polarized Vibrational Density of States of Polyacetylene from Incoherent Inelastic .Veutron Scattering".

J. L. Sauvajol, D. Djurado, A. J. Dianoux. N. Theophilou and J. E. Fischer', Phys. Rev. B 43. 14305 (1991).

17. "Compressibility of Solid $C_{60}$ ", J. E. Fischer, P. A. Heiney, A. R. IfcGhie. W. J. Romanow. A. M. Denenstein.

J. P. McCauley Jr. and A. B. Sinith IYI, Science 252, 1288 (1991).

.8. "Fluorinated Fullerenes", H. Selig, C. Lifshitz, T. Peres, J. E. Fiscier, A. R. IIcGhie, WV. J, Romanow.

J. P. McCauley Jr. and A. B. Smith III. J. Am. Chem. Soc. 113. 5475 (1991).

19. "Charge Stripping $C_{60}^{+}$". C. Lifshitz, M. Iraqi, T. Peres and J. E. Fischer. Rapid Comm. Mass Spec. 5. 238 (1091).

20. "Orientational Ordering Transition in Solid $C_{60}$ ", P. A. Heiney. J. E. Fischer. A. R. McGhie. W. J. Romanow.

A. M. Denenstein, J. P. McCauley Jr., A. B. Smith III and D. E. Cox. Phys. Rev. Lett. 66, 2911 (1991).

21. "Structure and Bonding in Alkali Mletal-Doped $C_{60}$ " O. Zhou. J. E. Fischer. N. Coustel, S. Kycia. Q. Zhu.

A. R. McGhie, W. J. Romanow, J. P. McCauley Jr. and A. B. Smith III, Nature 351, 462 (1991).

22. "Structure and Low Energy Dynamics of Solid $C_{60}$ ", J.R.D. Copley, D.A. Neumann, R.L. Cappelletti. IV.A. Kamitakiahara, E. Prince. N. Coustel, J. P. McCauley Jr., N.C. Maliszeivskyj, J.E. Fischer. K.MI. Creegan and D.MI. Cox. Proceedings of International Conierence on Neutron Scattering. Oxford UK - Physica B (to be rublished)

23. "Channel Structures in Alkali-Doped Conjugated Polymers: Broken-Symmetry Intercalation Lattices".

P. A. Heiney, J. E. Fischer, D. Djurado, J. Ma, D. Chen. II. J. VVinokur, N. Coustel. P. Bernier and F. E. Karaszi, Phy.s. Rev. B42, 2507 (1991).

24. "Structural Characterization of Oriented Polyacetylene Films Grown by the Liquid Crystal Method". N. Coustel. Y. Foxonet, J. L. Ribet, P. Bernier and J. E. Fischer, Macromolecules (December 1991).

25. "Percolation in Doped Conducting Polymers: Alive and W'el!!". J. Voit. N. Coustel. P. Bernier and J. E. Fischer. in Sipringer Series in Solid State Sciences (1991). 
26. "Pressure-Induced Staging Transitions in TiS2 Intercalation Compounds", O. Zhou. J. E. Fischer and K. S. Liang. Physs. Fiev. (in press).

27. "Intrachain Dynamics and Interchain Structures of Polymers: A Comparison of Polyacetylene, Polyethylene. Polynniline and (Poly)paraphenylene-vinylene". J. Ma. J. E. Fischer. E. M. Scherr. A. G. MacDiarmid.

II. E. Josefowicz, A. J. Epstein. C. Mathis, B. Francois. X. Coustel and P. Bernier. Phy's. Rev. (submitted).

28. "Solud ("ijo: Structure. Bonding, Defects and Intercalation", J. E. Fischer. P. A. Heiney. D. E. Luzzi and

D. E. ('ux, in Large Carton Clusters. American Chemical Society Symposium Series (to be published).

20. "Simhesis. Structure and Superconducting Properties of Single Phase $R b_{3} C_{50}$ : A New Convenient Method for the Preparation of $M_{3} C_{\text {ro }}$ Superconductors". J. P. McCauley Jr.. Q. Zhu. .V. Coustel. O. Zhou. G. Vaughan. S. II. I. Idzint. J. E. Fischer. S. W. Tozer. D. M. Froski, N. Bykovetz. C. L. Lin. A. R. McGhie. B. H. Allen. W. J. Romanow A. II. Denenstein and A. B. Smith III. J. Am. Chem. Soc. (subnitted).

30. "Electrochemical Lithium Intercalation in Solid $C_{B 0}$ ". Y. Chabre. D. Djurado. M. Armand. W. J. Romanow.

․ Coustcl. J. P. McCauley Jr.. J. E. Fischer and A. B. Smith III. J. Am. Chem. Soc. (submitted).

\section{G. PEI.SONNEL SUPPORTED BY THIS GRANT}

John E. Fischer, principal investigator

Otto Zhou. PhD student (9/8T - present. degree expected $9 / 92)$.

Andreas Eversbuch, PhD student ( $9 / 89-1 / 90$; dropped out).

Qing 7hu. PhD student (1/90 - present. degree expected 1995).

David Djurado. Postdoc $(2 / 88-5 / 8): 1 / 3$ salary from grant. balance from C.NRS).

David Vaknin. Postdoc (6 months ir.termittent. 1988-90).

Vicole Coustel. Postdoc (10/90 - 9/91).

William Romanow Jr.. summer student 1991 (Stanford University).

Brent Allen. summer student 1991 (Penn).

Nicolas Bytovetz. sabbatical visitor ( $8 / 91$ - 6/92; $1 / 4$ salary from grant, balance from Dept.)

II elen .Mertwoy, chemical technician ( $i / 91$ - present: $1 / 4$ salary from grant. balance from LRS.M).

Co salary charged to grant:

* Thandi Buthelezi, summer student 1990 Williams College (chenistry)

* Dacai Xie. Penn postdoc at B.iL reactor (5/89 - present)

* Claude Mathis. visitor 6-8/90 from Strasbourg, France

* Victoria Cajipe, visitor 6-12/00 from IPCM. Nantes. France

* Henry Selig, visitor $7-8 / 01$ from IIebrew University, Jerusalem

* Oleg Zharikov. NAS visitor 3-8/90 from USSR Acad. Sci.. Mloscow

* Andrey Palnichenko, ․AS visitor 3-8/90 from USSR Acad. Sci.. Moscow

* Patrict Bernier. frequent visitor from CNRS. Montpellier. France 

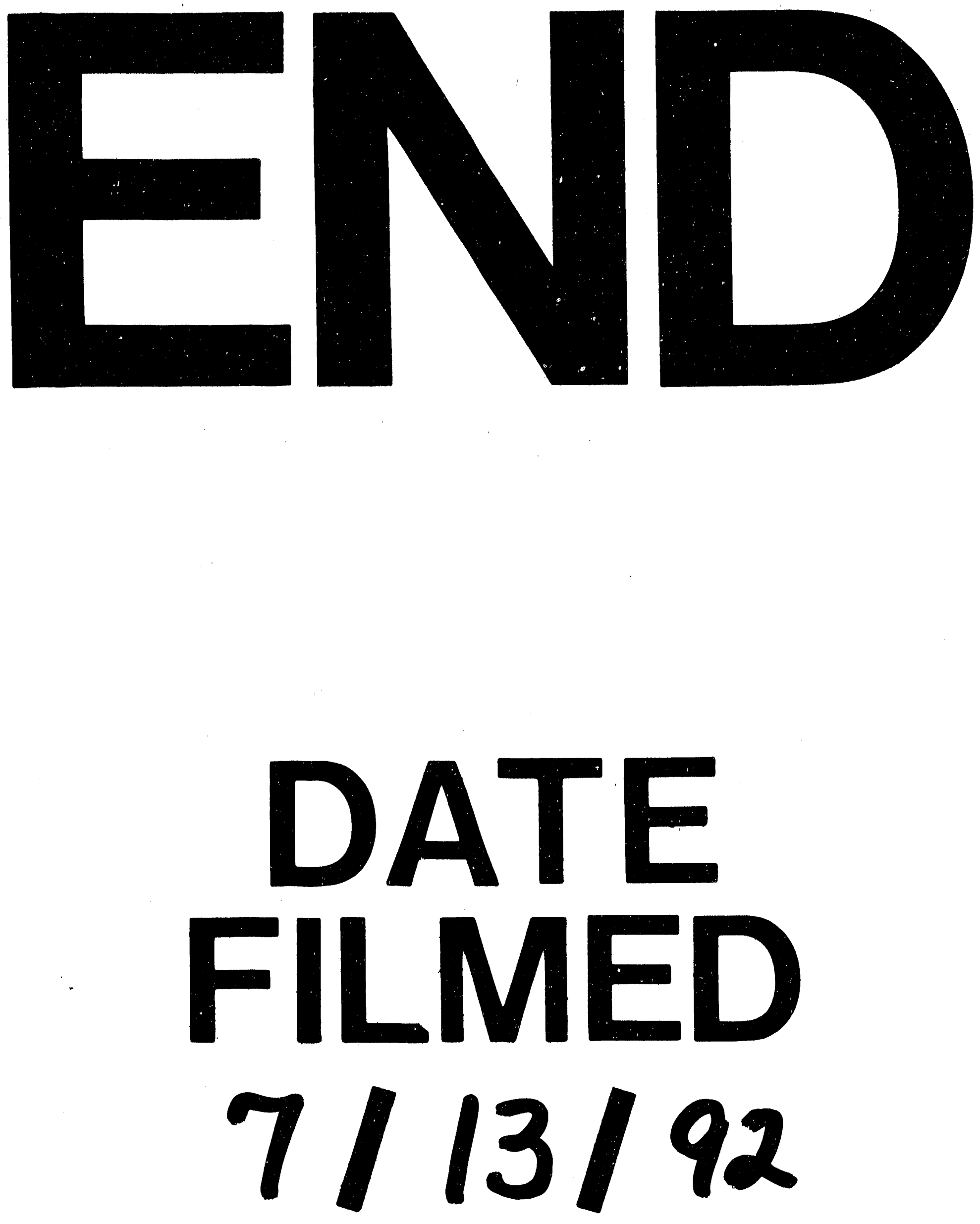
